\title{
LIVRES
}

\section{The penis}

\author{
A. HASHMAT \\ Lea et Febiger, Philadelphia, London, 1993, \\ 350 pages, 190 ., ill
}

Cet ouvrage en anglais comprenant sept sections passe en revue l'ensemble de l'anatomie, de la physiologie et de la pathologie du pénis, tant sur le plan diagnostique que thérapeutique. Il a le mérite d'une mise en page claire, d'illustrations abondantes et d'excellente qualité. Exhaustif, il aborde aussi bien la pathologie de l'adulte que celle de l'enfant et couvre de plus les traumatismes, les urgences et les problèmes dermatologiques. C'est en 1993, le livre de chevet indispensable de tout andrologue (M. Schouman).

\section{Manuel de laboratoire de l'OMS. Analyse du sperme humain et de l'interaction des spermatozoïdes avec le mucus cervical}

Traduction de Jacques Auger et Pierre JouanNET

Les Editions INSERM, Paris, 1993, 125 pages.

Cet ouvrage est la traduction française de la troisième édition (1992) du WHO Laboratory Manual for Examination of Human Semen and Sperm - Cervical Mucus Interaction. L'andrologue francophone, biologiste ou clinicien, dispose enfin d'un manuel simple qui rassemble la quasi totalité des moyens actuels d'exploration fonctionnelle de la fertilité masculine. L'objectif du groupe d'experts internationaux à l'origine des textes est avant tout de proposer des méthodes codifiées et standardisées, parfaitement reproductibles, et dont l'intérêt est non seulement d'améliorer le diagnostic et la thérapeutique mais aussi de permettre des études épidémiologiques et des recherches multicentriques.
Le chapitre consacré à l'examen du sperme est divisé en trois parties. La première partie décrit les méthodes constituant le minimun indispensable pour l'analyse du sperme. La deuxième partie décrit les explorations considérées comme facultatives. Enfin la troisième partie présente des méthodes encore considérées comme des outils de recherche.

Le chapitre traitant de l'interaction des spermatozoïdes et du mucus cervical est assez longuement développé, soulignant l'importance de l'étude du comportement des spermatozoïdes dans les voies génitales féminines.

Les différentes techniques utilisées sont précisées par des fiches placées en annexe.

Une bibliographie de 79 références donne accès aux principales publications à partir desquelles le manuel a été élaboré. (J.C. Czyba)

\section{Masculin-féminin ou la guerre impossible}

Maurice Auroux

Buchet/Chastel, Paris, 1993, 373 pages.

Cet ouvrage, destiné à un large public, se lit avec plaisir et facilité. Qu'on ne s'y trompe cependant pas, Maurice Auroux est un hospitalo-universitaire plein d'expérience et de subtilité et son texte plein d'humour est le fruit d'une solide réflexion appuyée sur plus de 300 références bibliographiques.

Professeur de Biologie du Développement et de la Reproduction (et membre de la SALF depuis ses origines) Maurice Auroux est connu pour ses travaux sur les méfaits de l'âge du père sur sa descendance.

Après avoir publié, il y a quelques années, chez le même éditeur, un essai sur “l'ambiguité 
humaine", l'auteur nous présente aujourd'hui un livre sur la différenciation sexuelle, ses aspects, ses mécanismes, ses défaillances, ses représentations idéologiques et sa finalité qui est la perpétuation de l'espèce.

Il nous est montré que la différenciation sexuelle qui s'opère pendant tout le développement, de la fécondation à l'état adulte, aboutit à la formation de deux individus différents mais complémentaires ("la clé et la serrure") pour l'exercice d'une très agréable sexualité forcément reproductrice si on n'y prend pas garde. Vérités premières ? Oui, mais énoncées par un biologiste qui sait bien que quelles que soient les idéologies il ne faut jamais perdre de vue hormones, organes et cerveau (sexualisé et sexualisant). L'auteur s'efforce de dégager les "constantes" qui caractérisent les attitudes et comportements masculins et féminins à travers l'histoire de l'humanité et dans toutes les sociétés, soulignant le rôle amplificateur des codes socio-culturels sur une différenciation biologiquement déterminée. Les discours machistes et féministes sont apréhendés avec une prudente mais efficace malignité. L“idéologie unisexe" est montrée comme un produit de l'imagination au service du désir de partager tous les rôles. L'imagination imagine et l'intelligence permet la réalisation des systèmes hors nature que l'intelligence a conçus". Certes, "l'homme s'est toujours écarté des conditions dites naturelles pour peu à peu, maîtriser la nature", et, d'autre part, son intelligence adaptative lui permet d'assumer les nouveaux rapports entre les deux sexes qui résultent à la fois de l'évolution des conditions socio-économiques et de la démocratisation. Mais, les systèmes conçus par l'intelligence peuvent être comme "des ressorts dans une boîte qui n'est pas faite pour eux : il faut sans arrêt faire attention qu'aucun ne s'échappe".

L'auteur conclut en dénonçant la confusion habituelle entre justice et égalité et, s'il plaide pour "un partage équitable des gratifications" entre les deux sexes, il demande qu'on veuille bien reconnaître l'évidence "qu'une femme est une femme et un homme un homme" (J.C. Czyba). 